\title{
Participatory Research with Older Adults with AMD: Co-Designing a SMART Diet Diary App
}

\author{
Lilit Hakobyan \\ School of Engineering \& \\ Applied Science, \\ Aston University, \\ Birmingham, B4 7ET \\ hakobyl1@aston.ac.uk
}

\author{
Jo Lumsden \\ School of Engineering \& \\ Applied Science, \\ Aston University, \\ Birmingham, B4 7ET \\ j.lumsden@aston.ac.uk
}

\author{
Dympna O'Sullivan \\ School of Informatics, \\ City University London, \\ London, \\ EC1V OHB \\ dympna.o'sullivan.1@city.ac.uk
}

\begin{abstract}
The global population of people aged 60 years and older is growing rapidly. In the UK, there are currently around 10 million people aged 65 and over, and the number is projected to rise by $50 \%$ in the next 20 years (RNIB, 2013). While ongoing advances in information technology (IT) are undoubtedly increasing the scope for IT to enhance and support older adults' daily living, the digital divide between older and younger adults $-43 \%$ of people below the age of 55 own and use a smartphone, compared to only 3\% of people aged 65 and over (AgeUK, 2013) - raises concerns about the suitability of technological solutions for older adults, especially for older adults with impairments. Evidence suggests that sympathetic design of mobile technology does render it useful and acceptable to older adults: the key issue is, however, how best to achieve such sympathetic design when working with impaired older adults. We report here on a case study in order to outline the practicalities and highlight the benefits of participatory research for the design of sympathetic technology for (and importantly with) older adults with impairments.
\end{abstract}

User-centred design (UCD). Participatory design (PD). Age-related macular degeneration. Older adults. Mobile assistive technology. Diet diary.

\section{INTRODUCTION}

In the UK alone, there are approximately 10 million people aged 65 or over, of which almost 2 million are living with sight loss (RNIB, 2013) - one of the most serious age-related health concerns among older adults (Congdon et al., 2004). The most common cause of sight loss in the UK is age-related macular degeneration (AMD); typically affecting people aged 50 and above, it impacts nearly one in ten of those over 80 (RNIB, 2013) and accounts for $16,000 \mathrm{blind} /$ partial sight registrations per year (Despriet et al., 2006). As a progressive, degenerative disease of the eye it severely affects the macula, located at the centre of the retina, which is vital for clear central vision (see Figure 1).

AMD substantially limits the independence of elderly patients as a result of the increased challenges associated with completion of daily activities and reduces their quality of life (Mitchell et al., 2008). Strategies to combat AMD are now focusing on prevention of AMD progression rather than expensive pharmaceutical treatments which are not universally effective.

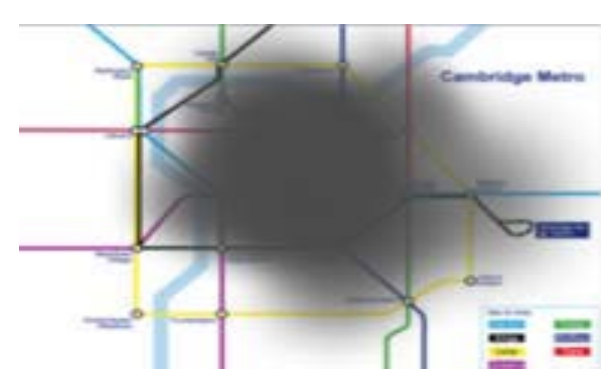

Figure 1:Example of how a subway map might be viewed through an eye affected by AMD [generated using www.inclusivedesigntoolkit.com].

There is evidence that there are links between dietary factors, AMD risk, and AMD progression (AREDS, 2001). Thus, it is considered important to encourage persons at risk to maintain a diet high in specific nutrients such as carotenoids (Chong, 2008).

While existing technologies (e.g., electronic diet diaries) could potentially be beneficial for delivering interventions to promote appropriate dietary intake, as highlighted by Figure 1 AMD presents a significant challenge in terms of the user interface (UI) design for such technology - a challenge which 
is further complicated by the degenerative nature of the disease. Recognising the importance of good nutrition and the challenges involved in designing for people with AMD, our goal is to develop a sympathetic, ability-reactive assistive mobile application (SMART) to support accurate and convenient diet data collection on which basis to then provide customised dietary advice and recommendations in order to help support individuals with AMD to mitigate their ongoing risk and retard the progression of the disease. We report here on our ongoing work, highlighting the design methods used to engage end users in our research and introducing the early proof-of-concept prototype of our proposed SMART application, as a case study for symathetic design of technology with and for older adults with disabilities. To date, we have engaged with our target users for the purpose of knowledge elicitation and design phases of our research, both phases having been previously reported at $\mathrm{BCS} \mathrm{HCl}$ conferences (see Hakobyan, et al., 2012; Hakobyan et al., 2013a). Here, we provide an overview of our overall experience of initiating and managing user participation in a research project of this nature following the guidelines suggested by Leung and Lumsden (2008), including how we addressed issues such as establishing relationships with communities and participants to determine the research context and secure representative end-user engagement. We also provide (a) a brief discussion of our practical findings with regard to our app design itself as an illustration of the richness of information elicited by, and value of, engaging in participatory research with older adults with AMD, and (b) a reflection on the (technical) challenges associated with attempting to build atypically-designed applications within the Android framework. We hope this will contribute to the growing body of research within $\mathrm{HCl}$ investigating how special needs user engagement within research projects is generally managed, and raise awareness about the contradictions that exist between UI design requirements as dictated by special needs target users and technical mobile development platforms catering to the masses.

\section{BACKGROUND}

As the field of $\mathrm{HCl}$ is increasingly exploring possibilities for enabling ageing and disabled populations to take full advantage of technological advancements to improve their quality of life, we have seen increased research focused on the involvement of special needs users within different stages of technology design/development in order to maximise its potential for impact and acceptance by such user groups (e.g., Lindsay, et al., 2012; Leung and Lumsden, 2008; Forbes, et al., 2009). Wobbrock, et al. (2011) advocate the philosophy of ability-based design, which encourages designers to refocus from users' disabilities to their abilities - that is, to focus on what users can do rather than work around what they can't. They have derived seven principles to support the application of this thinking which focus on the designers' stance (i.e., their focus on users' abilities), the interface (in terms of its adaptability and transparency), and the system (measuring performance, context and commodity).

Technologies designed specifically for the visually impaired have the potential to enhance their quality of life via improved autonomy and safety and sustained independence. Focusing on the abilities (e.g., sense of touch, auditory capacity, remaining vision) of the visually impaired has led to advances in technology specifically for this user group, including interactive maps using tactile and auditory output (Brock et al., 2012) and a novel exergame, Eyes-Free Yoga, using auditory-only feedback based on skeletal tracking (Rector et al., 2013). Although moves have also been made to effectively engage the visually impaired directly in the design of technologies for their use (e.g., via accessible haptic user interface design approaches for users with visual impairments (Kim et al., 2013)), inclusive methods for the design of technologies for this user group have not yet received sufficient attention, despite the opportunities for significant impact. When researching the design of novel technologies for the visually impaired, it is important to consider the heterogeneity of their capabilities in terms of the extent of their visual impairment and their ability to use whatever vision they may have. It is also imperative for designers to fully understand what it is like to live with the disability and this is typically best achieved by engaging in user-centred, ideally participatory, design. Despite this, in the case of $A M D$, persons suffering from the degenerative eye disease have not traditionally been directly involved in the design of technology to support their needs and abilities. They have been the focus of a study into handheld GUI-based computer interaction for older adults with AMD which identified that severity of the disease, design efforts and strategies, and contrast sensitivity were important indicators for successful iconic search using, and manipulation of, handheld computers by this user group (Leonard, et al., 2005). Beyond this, however, research into designing technology to match the abilities of this user group to date has been limited to a more general focus on desktop computers for the visually impaired rather than (mobile) assistive technologies for persons with AMD. Besides their challenges with focus-intensive tasks (as a consequence of the degeneration in central vision), individuals with AMD are also likely to suffer from other sensory and motor impairments (as a consequence of ageing) and, as such, find interaction with technology (and possibly participation in research studies overall) rather challenging. We have addressed these 'challenges' head on in our pursuit of technology to mitigate AMD 
risk and retard degeneration in older adults with the disease.

\section{OUR RESEARCH APPROACH}

Despite recognised benefits, prior research in the field of assistive healthcare technology development has highlighted that the inclusion of individuals with disabilities and domain experts in the design process is essential but not without its difficulties (Crabtree et al., 2005). One of the main reported issues is finding and recruiting representative participants (Allen et al., 2008). In an attempt to maximise our potential for success in terms of engaging with individuals with AMD in our research, we followed six out of Leung and Lumsden's (2008) seven guidelines (the last guideline being applicable to evaluation stages which we have not yet reached) recommended for effective inclusion of special needs users in the design process of assistive technologies. We introduce each guideline and discuss how we observed it in turn below. For more detail about our methods and results please refer to (Hakobyan, et al., 2012; Hakobyan, et al., 2013a; Hakobyan, et al., 2013b).

\section{"Work with Existing Support Organisations"}

Working with organisations that support individuals with the particular target disability is advocated as an effective means of overcoming potential difficulties associated with involving target users in research projects. At the onset of our project, we consulted domain experts (e.g., optometrists and ophthalmologists) to elicit their expert opinion on how to design our proposed technology and, most importantly, on how to engage individuals with AMD directly in the project. Interestingly, although we anticipated there were existing frameworks for involving AMD users in research projects in the field of ophthalmology, we learned that the notion of user participation in that field was very different to the democratised approach to their involvement we were proposing. Following this, we engaged with local community support groups for people with AMD, attending several of their meetings which allowed us to immerse ourselves within the community and start getting to know its members. Over a period of 2 months we attended 4 meetings where we had the opportunity to informally introduce ourselves and our project goals, participate in group activities, and to start to learn about our target users' condition and, accordingly, capabilities and limitations directly from the support network and its members. This allowed us to build a trusted professional relationship with individuals with AMD in an environment in which they were comfortable and to ultimately elicit their voluntary involvement in our research.

\footnotetext{
"Assess Target Users' and Domain Experts' Needs, Abilities, and Expectations"
}

To start learning about and, as such, better understand various aspects of our target users' lives beyond their medical diagnosis (in particular, how they cope with living with sight loss), to understand their experience with and attitudes towards (mobile) technology, to help us to effectively plan subsequent stages of our research in terms of being sympathetic to our potential participants'/users' abilities and needs, to enable us to determine the context and setting of our future activities, and to allow us to engage potential participants for our participatory design activities, we recruited 10 volunteers (nine people with AMD and one carer - all over 70 years of age) from the community support groups we attended for a series of focus groups. We ran these informal, small group discussions every few weeks at a venue convenient to the participants (generally at the same venue as their normal support group meeting or a nearby coffee shop to remove barriers to participation and put participants at ease). We established two groups, one which met three times and one which met four times over a period of 4 months. Each focus group session lasted no more than 2 hours and comprised 5 people plus a single researcher/moderator. All focus group sessions were audio-recorded: a verbatim transcription of each recording was subsequently generated for analysis.

Emerging from the focus group discussions was a realisation of the true extent of heterogeneity of individuals' capabilities, experiences of living with AMD, and, as a result, the significant differences in their needs in terms of acceptance of assistive technologies. As such, reflecting on this learning, we considered it essential to attain a true sense of 'being there' with representative participants, experiencing their daily life in an attempt to gain (a) a detailed appreciation of their daily coping strategies and what it is like to live with AMD, and (b) their technological needs so that we could ideally model their daily coping strategies in such a way as to be able to map relevant concepts into the design of our technology. To do this, we conducted a series of in-home observation sessions over the course of three months. We recruited 4 participants from the focus groups (one male and three female) who were particularly and eagerly engaged with the process and willing to participate in this next phase. In total, 6 observational sessions were conducted; the number of sessions conducted per participant was determined by availability and also by professional judgment as to whether additional sessions with the given individual would elicit new data (i.e., a judgement as to whether data saturation had been reached); each session lasted no more than an hour. Observations were kept very informal to ensure participants felt at ease: handwritten notes were taken, and the researcher engaged in discussion with each participant as befitted the situation. Finally, we engaged in a series of interviews with 
clinical experts to determine their expectations and opinions regarding our proposed healthcare intervention.

\section{"Choose a Design/Evaluation Technique and Analyse its Requirements"}

To empower our target end users to contribute as full and equal participants in the design process, we invited 4 already-involved individuals (comprising 3 with $A M D$ and a carer who also had AMD) to become integral members of our participatory design (PD) group - that is, they became full and active members of the design team for the purpose of democratic hands-on design of novel technology to support their healthcare and independent living needs. Comprising members of different ages, different stages of visual impairment, and different levels of IT literacy, we believe our participatory design team was representative of the heterogeneity of the AMD community whilst being ideally sized to encourage active participation. We selected these individuals on account of their by-now-established rapport with the researcher, their demonstrated keenness to contribute to our research agenda, and their comfort in interacting (as a consequence of growing interpersonal bonds) with each other. We adopted the PICTIVE (Plastic Interface for Collaborative Technology Initiatives through Video Exploration) participatory design process (Muller, 1992), as it utilises standard office stationary (e.g., Post-It notes, pens, paper, etc.) to create paper prototypes of a user interface design and so was considered most appropriate for our user group on the basis of its inclusive and non-intimidating format.

\section{"Adapt the Chosen Approach to be Sympathetic to the Target Users' Abilities" and "Attempt and Refine the Approach"}

Over a period of 5 months, participants attended 8 design meetings in order to directly contribute as experts in living with their condition to the design of our SMART application. The design sessions took place at the university, in a room chosen for ease of access and good lighting - room selection having been informed by reflection on the in-home observations. Return taxi-based transport was provided between participants' homes and the university to remove physical barriers associated with commuting and therefore participating. During the design sessions, participants were comfortably seated around a shared design surface on which they worked (see Figure 2). All sessions were recorded by a video camera (to which participants had consented); the area captured by the camera was delineated in blue tape (see Figure 2) on the design surface to ensure all relevant activities took place in view and to allow participants space to work 'off the record' if desired. Because the camera was not focused on them (but instead was on the 'working zone' in the middle of the table) participants essentially ignored it and so it permitted an accurate and detailed record of the participation without skewing the process.

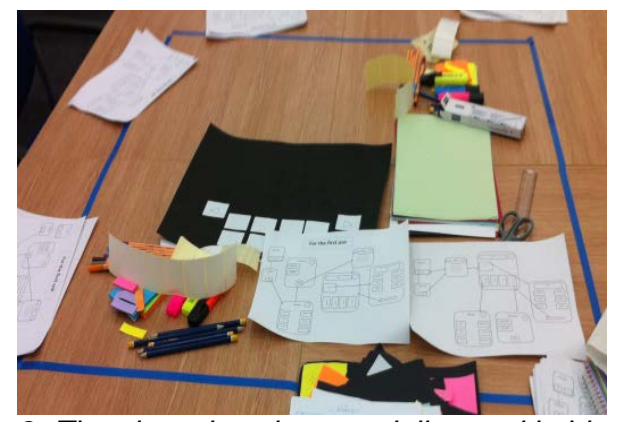

Figure 2: The shared workspace delineated in blue tape.

After each session, the researcher had the opportunity to reflect on the outcomes and observed participant contributions - reflection which was aided by the video record - and this reflection supported effective establishment of the research context/questions for the subsequent sessions. We had to give due accommodations to the way in which the sessions were conducted to account for (a) participants' individual abilities, and (b) the fact that this was a novel experience for the them. In summary, our accommodations and refinements included (for more detail see (Hakobyan, et al., 2013b; Hakobyan, et al., in press)): (i) adapting the PICTIVE method to include individual copies of the design to support individuals' visual viewing abilities as opposed to only relying on the central design artefact; (ii) making appropriate use of metaphors and pertinent tangible objects to assist participants in envisaging mobile assistive technologies, visualising their design ideas, and to encourage their creative thinking; (iii) using accessible, nontechnical language when providing explanations and guiding discussions; (iv) identifying and accommodating participants' comorbidity issues when administering the PD sessions; and (v), at all times, we established a friendly atmosphere in which the participants felt comfortable to work.

\section{"Clearly Communicate the Nature of Participants' Involvement"}

From consulting domain experts and attending the local community support group meetings it was apparent that the main reason people expressed for being reluctant to participate in research studies was a misconception that lab-based research essentially used people as experimental subjects rather than experts living with their condition. To address such misconceptions and, as such, fully benefit from their participation in our research, we needed to convince our participants, in both discussion and action, that we considered them as experts in living with their condition and that our research was entirely aimed at meeting their needs (rather than the other way around); our success in doing this not only allowed for rewarding and informative discussions, but it ultimately led to us being able to invite participants 
(as outlined above) to take part in the subsequent stages of our project, thus substantially easing the process of finding and recruiting participants for those stages. Given the potential issues of vulnerability associated with participants' capacity to read documentation associated with the study, in all phases of our study we have paid particular attention to valid mechanisms for fully informing them about the study and obtaining their consent to participate. To this end, all documentation (including consent forms and questionnaires) has been produced in various font sizes and distributed in advance of each phase of the study so that participants could turn to family/support workers to help them read the material and give them a chance to ask any questions before consenting to participate; in addition, we provided detailed verbal explanation when handing out such documentation.

\subsection{Reflections}

Here, we briefly reflect on the main findings of the research activities outlined above; as previously noted, for detailed information we would direct interested readers to (Hakobyan, et al., 2012; Hakobyan, et al., 2013a; Hakobyan, et al., 2013b).

In general, by following the guidelines, we feel that it has been possible to minimise challenges associated with enabling target users to effectively participate in the design and development of technology; by taking appropriate steps at the beginning of our participatory research agenda, we gained the trust and commitment of members of an otherwise reticent population, enabling us to empower them to be a strong driving force behind the success of our design activities.

Despite industry advances in making mobile phones more accessible for the visually impaired, it was apparent from our focus group discussions that such devices are not yet fully accessible or acceptable to people with AMD. Despite this, however, participants' enthusiasm towards current technology, even from this early stage, was encouraging and promising: of specific note was the enthusiasm with which participants viewed current technological devices in terms of what they can offer individuals with $A M D$ and the potential for such technology to enhance individuals' independence and quality of life - for example:

\footnotetext{
"Technology has gone on and on and we are behind and now we are in a position where technology can help us".
}

Their positive interaction with computers (for those participants who owned computers) demonstrated that older adults with AMD can and will use technology if the potential benefits of such use are easily understood and appreciated. We noted a number of prominent trends or commonalities across all observed participants in terms of their living arrangements with respect to accommodating their visual deficiencies. These included having welllit and simple interior design, light-coloured walls, a lot of lighting in every room (an arrangement that was, upon reflection, echoed when choosing the setting for our PD sessions as already mentioned). Furthermore, participants typically kept their homes very tidy, organised and, most importantly, kept things in convenient locations. These living arrangements identified an imperative need for intuitive and consistent design which also lacked visual clutter so that participants could rely on their memory if and when necessary (reflections on these observations are evident in our UI design) and it is interesting that the desired reliance on memory is in direct contradiction of the recognition rather than recall mantra to which we typically conform.

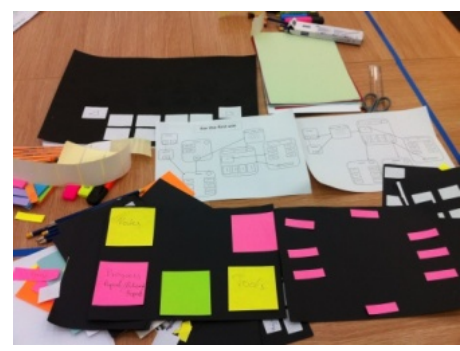

Figure 3a: Participants' arrangements of the UI components.

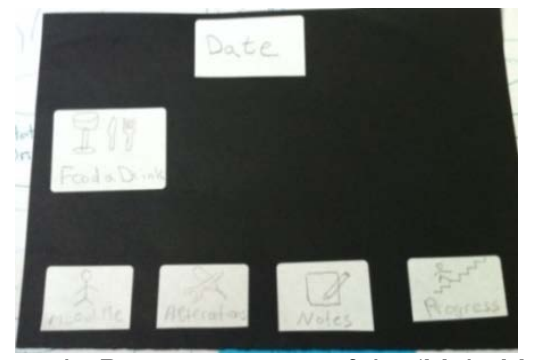

Figure 3b: Paper prototype of the 'Main Menu'.

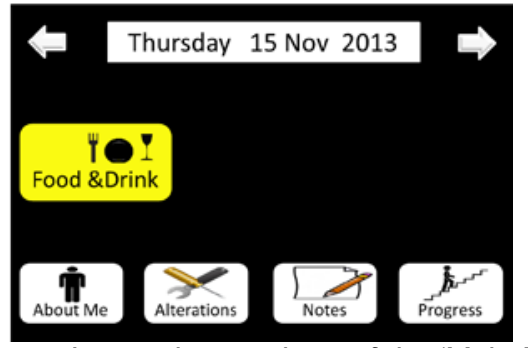

Figure 3c: Interactive mock-up of the 'Main Menu' created by the researcher using PowerPoint.

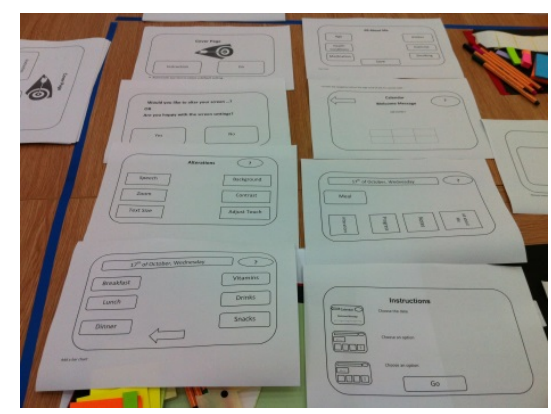


Figure 3d: Sketches of some of the screens created by the researcher.

In general, our PD approach proved successful at encouraging creative design thinking and inclusive participant contribution, regardless of level of visual impairment and computer literacy. While the primary outcome of our PD sessions was the specification of the UI for our SMART application in paper prototype form (see Figures 3a-d for an illustration of how the paper prototypes evolved during the process), results from our participatory work would also suggest that our involvement of participants has drastically altered, in a positive way, their opinion of research and their ability to contribute in a meaningful way to research of benefit to them.

During the last PD session, we briefly reviewed participants' thoughts on and experience of participating in the study process; we also conducted individual interviews at participants' homes 3 months after the final PD session to encourage participants to comment on the process with the benefit of hindsight (for a comprehensive discussion of both our reflections and those of our participants, see (Hakobyan, et al., 2013b)). Our aim was to ask for participants' feedback on their experiences of participating in our research study and the UCD methods used (i.e., from focus groups to design sessions), and to assess their opinion of the end design of which they had stakeholder ownership. Overall, participants' feedback indicated their delight and satisfaction in taking part in all aspects of the research study; they all also demonstrated a willingness (in fact keenness) to continue with the process:

\section{"It was challenging, thought provoking and exciting! I would happily be involved in future projects of this nature".}

To assess the validity of our prototype design beyond our PD participants, we attended a local community support group for people with AMD and asked volunteers - who had not previously been involved in any phase of the project - to provide informal, formative feedback on the higher-fidelity version of our prototype (e.g., Figure 3c). The PowerPoint mock-up was shown on a laptop, but participants had also the opportunity to 'see' and 'experiment' with a Samsung tablet; 6 participants volunteered to participate. In general, all six participants found the command/option naming and the design and use of icons to be 'clear', 'selfexplanatory' and 'straight-to-the-point'. One participant explained that the 'clever' design and use of the icons would eliminate the need to read labels and, as such, make the interaction more 'enjoyable'. Furthermore, all agreed that the placement of the interface components was suitable, and offered no alternative suggestions. Participants did not actually comment on the hardware choice, but agreed that they would use similar devices as long as they could see the interface and its components.

\section{DEVELOPING OUR SMART APP PROTOTYPE}

On the basis of our PD activities to date, we dismissed standard smartphones as our hardware choice, and agreed to focus on tablets instead. Further, we decided to develop the application for the Android platform in part because of its applicability across a range of devices and manufacturers, supporting greater ultimate choice in device size and price, etc.

The development of SMART comprised the development of a novel UI based on the designs generated from our PD sessions, and the development of a computational engine (back-end) that included the creation of a simple food ontology (that is, an abstract model of the different types of foods available together with their nutritional information and the daily recommended intake) using nutritional information sourced from the Macular Society (2014) and US Drug and Administration (2014) online resources, and the development of a rule engine that combines information from the ontology with data from user profiles (e.g., food preferences, medical condition, and age) and captured dietary data (i.e., daily food entries) to generate individualised recommendations. We focus, primarily, in the remainder of this discussion on the $\mathrm{UI}$ aspects of the development.

Upon creating an account users are presented with the option of conducting a simple vision test using an Amsler Grid (a test used for detecting macular degeneration - http://www.armd.org.uk/amsler.htm) for the purpose of personalising the layout of the UI based on measured visual acuity. The Amsler Grid looks like graph paper, with dark lines forming a square grid (see Figure 4). While staring at a dot in the centre of the grid, the users look for wavy lines and missing areas of the grid to indicate (by touching those areas) the boundaries of their blind spot; by measuring this, we can avoid placing UI components where we know users will not be able to see them easily. Although we use this information to personalise the interface to start with, users will always have the option of manually 'altering' the settings and the system itself monitors user interaction to auto adjust layout over time. In developing the proof-of-concept application we obviously tried to remain as true as possible to the design rendered by our participants via the PD sessions. This was not, in many cases, as straightforward as initially anticipated since our design concept typically 'broke' established design guidelines or norms and Android recommendations meaning that we had to find programming workarounds. 


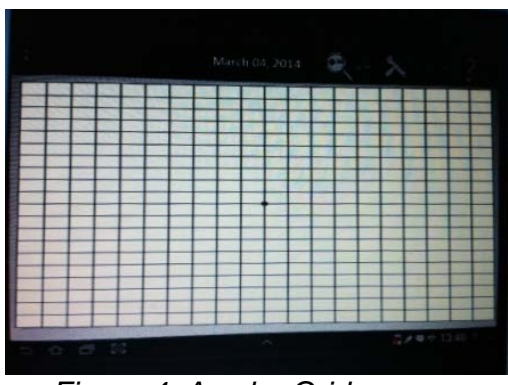

Figure 4: Amsler Grid screen.

For example, whilst the Android action bar supports consistent navigation, and has the capacity to reduce clutter by providing an action overflow for rarely used actions, we had to 'bend' Android's rules for this action bar to achieve the atypical design required for our app - for instance, Android design guidelines strongly recommend placing only icons on the action bar whereas we needed, based on our participants' preferences and requirements, to include both iconic and textual representations of functionality.

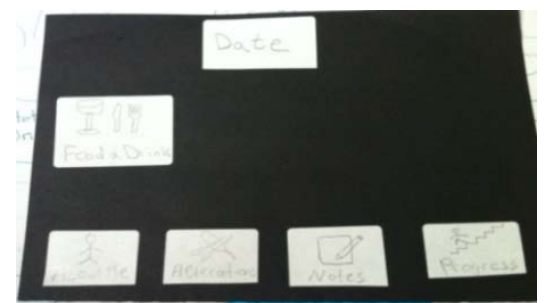

Figure 5a: The paper prototype of the 'Main Menu screen
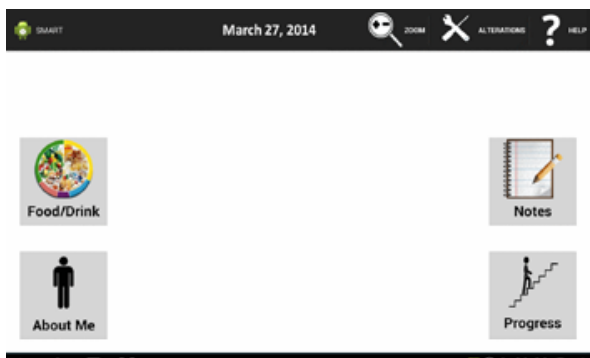

Figure 5b: 'Main Menu' screen as built

Figure $5 \mathrm{a}$ shows our participant-designed 'Main Menu' screen and Figure 5b shows its instantiation when implemented in Android. The options (or action buttons) that we have placed on the action bar include the 'Screen Name' for identifying user location, 'Today's Date' for viewing the calendar and selecting dates for food entry, 'Zoom Magnifier' for enlarging what appears onscreen, an option 'Alterations' for altering or making changes to the screen and personalising it, and a 'Help' option for viewing brief instructions.

The 'Alterations' option/screen (see Figures $6 a$ and 6b) facilitates personalisation of the app - that is, it enables users to individually tailor the application to better serve their needs and maximise application accessibility. From our fieldwork and design activities we learned that a black background was most beneficial to those users with the worst vision, whereas users with better eyesight preferred white background. Thus the option to change background was a core requirement. Similarly, users have the option to change text size and enable text-tospeech. A zoom magnifier is available here as well as on the Action Bar; this will be evaluated with participants to determine which option is preferred.

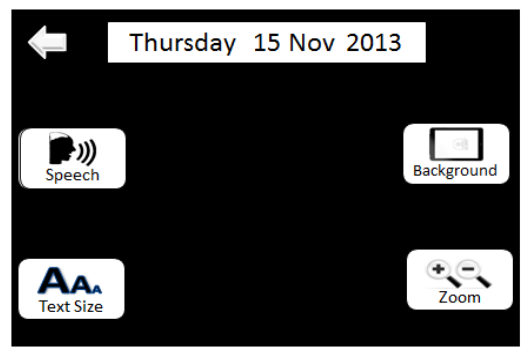

Figure 6a: Interactive mock-up of the 'Alterations' created by the researcher using PowerPoint.

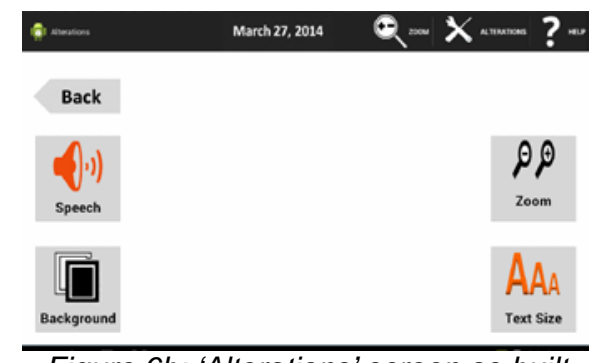

Figure 6b: 'Alterations' screen as built

Another key aspect of personalisation is providing customised dietary advice and recommendations to empower people with AMD to make informed dietary choices. To achieve this, users record information about their medical condition, disliked foods (in order to avoid inappropriate or unwelcome recommendations and hence maximise compliance), level of exercise and number of cigarettes smoked daily (both are taken into account when providing customised recommendations) (see Figures 7 and 8 for examples).

Users record (see Figure 9) their daily intake of food as meals, snacks and drinks, and also have an option to record intake of vitamins (such information also being taken into account when providing customised recommendations).

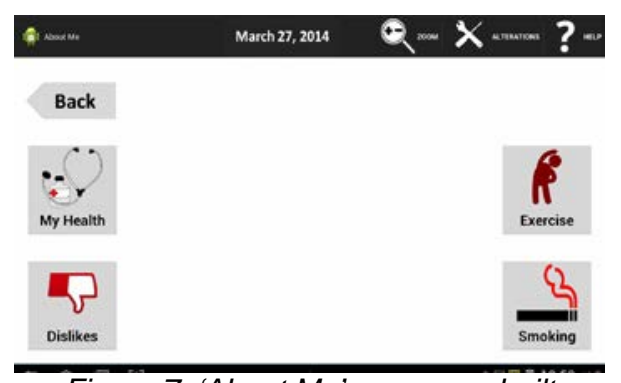

Figure 7: 'About Me' screen as built 


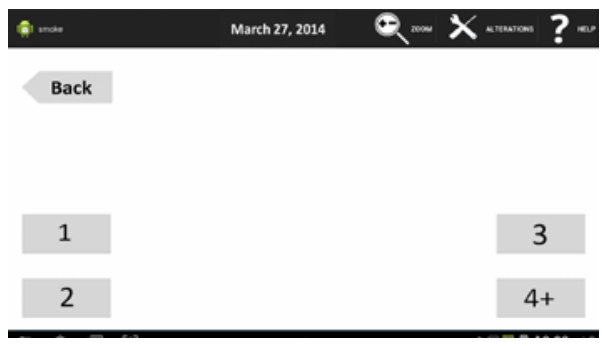

Figure 8: An example of how users would select the number of cigarettes smoked

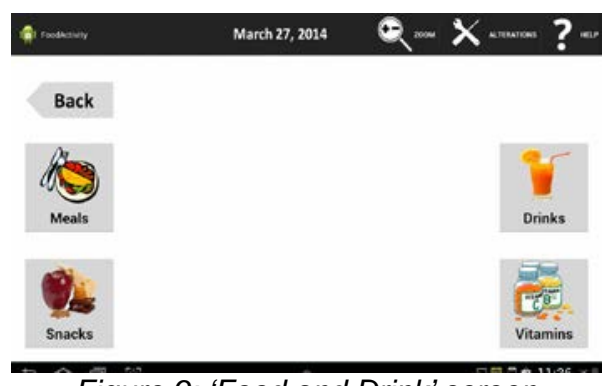

Figure 9: 'Food and Drink' screen

Additionally (see Figure 5b), users have a 'Progress' option for viewing their progress made in terms of adherence to dietary recommendations, and for accessing their recommendations (it is important to note that users will be automatically provided with customised daily dietary advice); and a Notes' option to 'store' their 'ideas' and thus support their memory.

\section{DISCUSSION}

In their recent review of how people are involved in design and participate in research within $\mathrm{HCl}$, Vines et al. (2013) consider three main goals motivating such participation: sharing control; sharing expertise; and inspiring change. In order to unpack aforementioned concerns as to how user participation is initiated and managed, and to analyse our own practice (including reflection on the resulting design of our SMART application), we structure our discussion here around the three main goals.

\section{Sharing Control}

As already noted, by adopting a participatory design method we hoped to empower older adults with AMD - that is, to make them feel in control and able to contribute to the design work without prior technical knowledge or expertise. This has resulted in a paper prototype design that reflects their views and needs and an implemented system that mirrors this as closely as possible, despite the standardised platform-imposed design guidelines and implementation hurdles (Android Developers, 2014). Although, in addition to very comprehensive design guidelines for standard apps, Android includes several features that support access for users with visual impairments (e.g.,'TalkBack' - a pre-installed screen reader service), Android does not encourage major deviations in user interface design itself. To accommodate and remain true to our participants' (and hence people with AMD in general) views, needs and opinions, we had to disregard some of Android's standard conventions or guidelines. For instance, as already noted, the guidelines encourage the use of standard, unlabelled icons in the action bar: we found, from our design sessions, that icon designs and naming conventions used within current applications were not consistent with our participants' mental models based on their life experiences, familiar environments and use of everyday objects and such served no useful purpose for them. Hence, not only did our PD team design our icons, but it also agreed that a simple label should always accompany each icon in order to greatly improve the acceptability and usability thereof, thus placing our participants more in control.

Furthermore, to achieve our participant-derived design, we had to ignore Android's recommended use of non-obvious interface elements, nested menus, reliance on long touches and hardware menu buttons when converting our paper prototype into a proof-of-concept application. This led us to question the derivation of the Android guidelines/norms, and their applicability beyond a 'normalised' user group. Other issues associated with instantiating the design in Android (e.g., the restricted placement of pop-up menus given that they are anchored to the base class of Layouts in Android) led us to reflect on the extent to which the final stage of development is strangled by technology norms such that control placed in the hands of the users during design ebbs away when faced with the practicality of delivering a working system. Nothing that we were trying to achieve was theoretically difficult, yet the framework imposed by Android made achieving an atypical, well-informed yet aesthetically 'unusual' design a struggle resulting in some compromises and leading us to question the extent to which special needs users' control extends to the delivery of a system.

\section{Sharing Expertise}

The mutual educational nature of our participatory design and fieldwork activities has been of crucial importance for the endurance and success of our project. Our objective for adopting UCD - and, in particular, participatory design - approaches was to learn about the needs of users with AMD, to appreciate the implications of designing for this user group and to understand how these can encourage (or hinder) technology use. Despite their personal challenges, our participants invested considerable time and effort in learning new skills; equally importantly, they taught us a great deal about their needs, experiences and expectations. In addition to the exchange of knowledge between the participants and the researcher, participants taught each other and delighted in each other's progress 
(i.e., when one participant agreed to help another participant 'operate' her mobile phone and socialise more). The overall experience of participating in our research study was truly an inspiring and encouraging experience for our participants; they were proud of being part of a research team - a noticeable change in opinion regarding research based on their prior experience.

\section{Inspiring Change}

By directly integrating participation of older adults with visual impairments (i.e., AMD) in the design process for assistive technology to support their needs, we anticipated supporting the establishment of a deeper and more valid understanding of their needs on our part, and contributing to influencing the success of technological development in terms of technology acceptance (including increased confidence in the use of technology) and ultimate impact (both in terms of improving peoples' lives and affecting future technology design).

For the ongoing success of this application, we will need to prove its capacity to positively affect behaviour change. Our design activities to date have highlighted participants' preferences for the practical motivational aspects associated with such change they agreed that beneficial feedback (e.g., customised recommendations) would motivate them to adhere to the dietary advice and recommendations and we have therefore benefitted from being able to encapsulate manifestations of psychological models such as the Theory of Planned Behaviour (Ajzen, 1991) into the practical design to maximise the potential to support individuals with $A M D$ in changing their dietary behaviour. We intend to evaluate this with target users during our upcoming longitudinal field evaluations.

\section{CONCLUSIONS}

We hope our case study has demonstrated how appropriate engagement with the user community can overcome challenges involved in engaging older adults with impairments in research projects and, consequently, yield invaluable results. The tangible results (i.e., our proof-of-concept application and elicitation of a comprehensive set of requirements) of our commitment to and wholehearted engagement in participatory research is clear evidence of the significant positive impact of directly involving target users in the design process, despite the perceived difficulty of so doing based on their (dis)abilities. The majority of our design findings would certainly not have been uncovered without the direct involvement of target users.

We are about to engage in longitudinal field evaluations wherein persons with AMD will use the device for an extended period of time in order to allow us to fully assess the acceptability to and impact of the system on this user group. In particular, our evaluation will aim to address the following research questions: 1 ) Will people use the diary?; 2) How do they use the diary?; 3) How effective is the UI adaptability and personalisation based on visual ability in terms of usability and acceptance?; and 4) Do users improve dietary behaviours in line with advice and recommendations?. While we have demonstrated why it is critical to engage older adults directly in the design and development of novel assistive technologies, we hope our evaluation studies will further validate our participants' design ideas in terms of the tool's generalisability and acceptability across more members of the AMD community. For now, we hope that this case study contributes in a positive and practical way to the growing body of evidence that older adults with impairments can be effective co-designers of technological solutions to meet their needs and abilities.

\section{ACKNOWLEDGEMENTS}

We would obviously like to thank all our AMD participants for their enthusiastic and invaluable involvement. This work has been supported in part by Aston Research Centre for Healthy Ageing (ARCHA).

\section{REFERENCES}

AgeUK, (2013) Age UK information guides and factsheets.http://www.ageuk.org.uk/publications/ age-uk-information-guides-and-factsheets/ (Accessed Mar 08, 2014).

Ajzen, I. (1991). The theory of planned behavior. Organizational Behavior and Human Decision Processes, 50(2), 179-211.

Allen, M., et al. (2008) Involving domain experts in assistive technology research. Universal Access in the Information Society 7(3), 145-54.

Android Developers, (2014) Design Principles. https://developer.android.com/design/getstarted/principles.html (Accessed Mar, 02, 2014)

Brock, A., et al. (2012) Design and user satisfaction of interactive maps for visually impaired people. Computers Helping People with Special Needs, Springer Berlin Heidelberg, 7383, 544-551.

Chong, E.W., et al. (2008) Dietary omega-3 fatty acid and fish intake in the primary prevention of age-related macular degeneration: a systematic review and meta-analysis. Arch Ophthalmol. 126, 826-33.

Congdon, N., et al. (2004) Causes and prevalence of visual impairment among adults in the United 
States. Archives of Ophthalmology,113, 477485.

Czaja, S., et al. (2006) Factors predicting the use of technology: findings from (create). Psychol Aging, 21(2), 333-352.

Despriet, D.D., et al. (2006) Complement factor $\mathrm{H}$ polymorphism, complement activators, and risk of age-related macular degeneration. JAMA 296, 301-309.

Crabtree, A., et al. (2003) Designing with care: Adapting cultural probes to inform design in sensitive settings. In Proc. OzCHI'03, Brisbane, Australia, Nov 26-28, 4-13.

Forbes, P., et al. (2009) Dundee user centre: a space where older people and technology meet. In Proc. Conference on Computers and Accessibility, Orlando, Florida, USA, October 2529, 231-232.

Hakobyan L., et al. (2012) Understanding the ITrelated attitudes and needs of persons with agerelated macular degeneration: a case study. In Proc. of BCS-HCl'12, Birmingham, UK, Sept 1214, 239-244.

Hakobyan L., et al. (2013a) Designing a Mobile Assistive Application with and for Older Adults with AMD: A Case Study, In Proc. of BCS-HCl '13, London, UK, Sept 9-13.

Hakobyan L., et al. (2013b) PICTIVE Participatory Design Process with Older Adults with AMD. In Proc. of iHCl '13, Dundalk, Ireland, June 12-13.

Hakobyan, L, et al. (in press) Older Adults with AMD as Co-Designers of an Assistive Mobile Application. IJMHCl. accepted; in press.

Kim, H. N., et al. (2013) Accessible haptic user interface design approach for users with visual impairments. Universal Access in the Information Society, 1-23.

Leonard, V. K., et al. (2005) An exploratory investigation of handheld computer interaction for older adults with visual impairments. In Proc. of Conference on Computers and Accessibility. Baltimore, USA, 12-19.

Leung, R. \& Lumsden, J. (2008) Designing Mobile Technologies for Individuals With Disabilities. In Handbook of Research on User Interface Design and Evaluation for Mobile Technology, Information Science Reference, Hershey, USA, 609-623.

Lindsay, S., et al. (2012) Empathy, participatory design and people with dementia. In Proc. CHI'2010, Austin, Texas, May 5-10, 521-530.

Mitchell, J., et al. (2008) The MacDQoL individualized measure of the impact of macular degeneration on quality of life: reliability and responsiveness. American journal of ophthalmology 146(3), 447-454.

Muller, M. (1992) Retrospective on a year of participatory design using the PICTIVE technique. In Proc. CHI'92, Monterey, California, June 3-7, 455-462.

Rector, K., et al. (2013). Eyes-free yoga: an exergame using depth cameras for blind \& low vision exercise. In Proc. ASSETS'13, Washington, USA, October 21-23, 12.

RNIB, (2013) Key Information and Statistics. http://www.rnib.org.uk/aboutus/Research/statistic s/Pages/statistics.aspx. (Accessed Mar 02, 2014).

Softpedia, (2012) Personal Health and Diet Manager. http://handheld.softpedia.com/ get/Health/Nutrition/Personal-Health-DietManager-4488.shtml. (Accessed 13 Mar, 2014).

The AREDS Research Group, 2001, A randomized, placebo-controlled, clinical trial of high-dose supplementation with vitamins $\mathrm{C}$ and $\mathrm{E}$, beta carotene, and zinc for age-related macular degeneration and vision loss: AREDS, Archives of Ophthalmology 119(10), 1417-1436.

The Macular Society. (2014) Nutrition. http://www.macularsociety.org/about-macularconditions/Nutrition (Accessed Mar 06, 2014).

US Food and Drug Administration. (2014) Protecting and promoting your health. http://www.fda.gov/ForConsumers/ConsumerUp dates/ucm244206.htm (Accessed Mar 02, 2014).

Vines, J., et al. (2013) Configuring participation: on how we involve people in design. In Proc. CHI'2013, Paris, France, Apr 27 - May 2, 429438.

Wobbrock, J. O., et al. (2011) Ability-based design: Concept, principles and examples. ACM Transactions on Accessible Computing, 3(3), 127. 